\title{
Beiträge zu einer kritischen Ausgabe des vatikanischen portugiesisohen Liederbuches.
}

Mit einer philologischen Arbeit beschäftigt, sah ich mich veranlafst, das vatikanische portugiesische Liederbuch durchzulesen, wobei sich mir Gelegenheit darbot, die von Braga gemachte kritische Ausgabe dieser Liedersammlung zu untersuchen. Dadurch bin ich zur Erkenntnis gekommen, dafs Braga's Werk das gute, welches es enthält, den Monaci's Ausgabe begleitenden unschätzbaren Anmerkungen, den Konjekturen von Coelho, und den Ausgaben von Moura und Varnhagen zu verdanken hat, im übrigen aber als eine sehr oberflächliche Arbeit zu bezeichnen ist. Denn Braga behält nicht die in der Handschrift befindlichen alten Formen mancher Wörter bei '; weicht von mehreren, von Monaci und Coelho vorgeschlagenen, oder in den Trovas e Cantares befindlichen richtigen Lesarten $a b^{2}$; lärst verschiedene Stellen in der Handschrift unverbessert, von denen entweder Metrik, oder Grammatik, oder der Gedankengang beweisen, dals sie fehlerhaft sind ${ }^{3}$; wo die Handschrift, wenn man keine Rücksicht auf den Zusammenhang und die Sprachrichtigkeit nimmt, auf zweierlei Weise gelesen werden kann, wählt er manchmal gerade diejenige Lesart, welche Sinn oder Grammatik ausschliefsen 4; für die fehlerhaften Stellen schlägt er zum öftesten Lesarten vor, welche entweder der Grammatik, oder der

- Braga schreibt perco für perço (immer so), perca für perça (immer so); como, und sogar $(63 / 5,867 / 8,1004 / 10,1139 / 5)$ aufs auffallendste com' $e$ für come, a tal f. atal (immer so, $748 / 5$ ausgenommen), a tanto f. atanto $(22 / 8$, $623 / 6)$, a tam f. atam (101/2, 787/12, 1073/12), salyva f. sayva (1017/13), 0 cajon f. ocajon (96/22), verso f. vesso (1088/53), sanar f. saar und sanou f. saou (1006/10 und 23), diabo f. diaboo (70/9), la f. laa (78/22), soub' $i$ f. soubi $(47 / 9,129 / 2)$, doas f. dras $(631 / 8,14,18)$, sirva f. servha $(370 / 11)$, dormo f. dormho (582/7), dorma f. dormha $(623 / 4)$, boa f. boa (oft, z. B. 49/12, 83/9, $89 / 3,94 / 13,100 / 14,101 / 7,456 / 8)$, u. a.

2 Man sehe weiter unten meine Anmerkungen zu 3/7, 12/7, 14/10, 18, 20, 16/13, I $7 / 13,18 / 9,26 / 2,33 / 19,35 / 2,48 / 14,56 / 12,251 / 4,428 / 4,542 / 15$, $583 / 8,585 / 8,625 / 16,667 / 13,733 / 2,793 / 18,837 / 15,916 / 9$.

3 S. $2 ! 7,15 / 6,16 / 10,12,25 / 6,87 / 3,100 / 2,113 / 4,260 / 2,300 / 4,312 / 10$, $329 / 5,444 / 12,494 / 3,543 / \mathrm{l}, 545 / 4,548 / 14,553 / 8,579 / 8,585 / 5,627 / 5,733 / 2$, $763 / 1,887 / 13,1047 / 12$.

+ S. 36/16, 40/10, 92/3, 120/9, 202/20, 216/6, 225/14, 289/11, =97/15, $301 / 5,309 / 21,328 / 7,353 / \mathrm{I}, 457 / 18,493 / 5,529 / 15,555 / 13,559 / 3,560 / 5,6$; $656 / 14,698 / 3$ : 
Netrik, oder des Zusammenhanges, oder sogar des Reimes wegen 1 , (und nicht selten aus mehreren von diesen Gründen zugleich)2, nicht anzunehmen sind, ja bisweilen erfindet er ganz ungeheuerliche, sinnlose $\cdot$ Wortgebilde ${ }^{3}$, und endlich vielmals setzt er sogar an Stelle der vollkommen verständlichen, fehlerlosen Lesart der Handschrift eine falsche, entweder den Sinn entstellende oder den Vers störende. ${ }^{4}$

$\mathrm{Da}$ die wirklich kritische Ausgabe des vatikanischen Liederbuches demnach noch $\mathrm{zu}$ veranstellen ist, sei es mir erlaubt, dazu einige bescheidene Beiträge zu liefern.

I/5 Ich lese: vedes que sandic' [ = welch eine Torheit] $e$ que gran loucura. Vgl.: vedes que mal $457 / 18$. Wie es aus $Z$. I hervorgeht, hält der Dichter nicht sich selbst für unsinnig, sondern diejenigen, die ihn nach dem Namen seiner Geliebten fragen.

Braga: vedes que sandec' é qu' e' gr. l.

2/7 Um der metrischen Richtigkeit willen ist die Lesart der Tr. e C.: pud' eu y al fazer (oder lieber pudi eu al fazer) anzunehmen.

Br. behält das handschriftliche pud' eu al fazer bei.

2/ro Vielleicht: mays per com' eu despois $m$ ' end' achei mal.

Br.'s Lesart: mays per com' eu d. m' eu a. m. ist grammatikalisch unstatthaft.

3/7 Man lese cuydei, wie die Metrik, der Zusammenhang und der Vergleich mit Z. 2 und 12 anzeigen, und wie es sich in $T r . e$ $C$. findet. Augenscheinlich ist das handschriftliche cuydedes ein Fehler des Abschreibers, welcher die unmittelbar folgende Partikel des zwcimal gesetzt hat.

$\mathrm{Br}$. behält cuydedes bei.

$7 / 3$ Ich interpungiere und lese: non vi; mais (aber), poys.

Br.: non vi mays; pois.

$7 / 4 \mathrm{Da}$ das Subjekt des guisou, auf welches sich das Fürwort el Z. 9 bezieht, nicht ausgelassen sein kann, so lese ich: Deus guisou, ja 'gora verei.

Br.: Guisou, já agora verei.

7/9 Lies: vivo (oder vive); mays (aber).

Br.: vyv'o mays.

12/7 L.: Aly hu eu d' ela quiley os meus, wie sich in Tr. e C. findet.

Br.: Aly hu d'ela $q$. os m.

IS. $2 / 10,17 / 8,20 / 9,25 / 3,66 / 1,74 / 2,81 / 17,89 / 9,114 / 2,184 / 9,196 / 13$, $246 / 5,8,10,523 / 1,531 / 3,538 / 5,540 / 12,547 / 2,550 / 7,621 / 1,647 / 13,663 / 7$, $675 / 8,704 / 14,909 / 17,921 / 18,981 / 14$.

2 S. $28 / 1,33 / 11,136 / 17,279 / 7,450 / 18,514 / 7,599 / 2,620 / 20,850 / 6$.

${ }^{3}$ S. $22 / 7,8,41 / 24,64 / 13,252 / 3,335 / 8,566 / 26,029 / 1,900 / 5$.

4. S. $17 / 14,17,20 / 3,14,160 / 13,196 / 16,253 / 7,260 / 3,298 / 1,300 / 9$, $354: 2,362 / 7,380 / 4,382 / 2,393 / 8,445 / 14,457 / 17,463 / 2,4,5,482 / 15,495 / 7$, $498118,501 / 2,531 / 3,531 / 3,545 / 8,560 / 19,598 / 7,632 / 8,663 / 28,697 / 12$, $700 / 9,704 / 14,771 / 5,11,16,818 / 10,822 / 15,19,20,916 / 4,11,1013 / 1$, $1100 / 4,5$. 
I4 Mit Monaci mufs man Z. 18 coices, wie es der Vergleich mit Z. 26 fordert, lesen, Z. 1o averey, Z. 20 Juyao, wie es die Metrik verlangt.

Br.: (18) çocos, (10) eu ey (20) vylao.

15/4, 5 Ich lese und interpungiere: sempr' ouv', e poys Deus quer assy, que. In der alten Sprache ist die erste Person des Perfectums ouvi, nicht ouve (ebenso pudi, soubi u. s. w.). Das e poys erkläre ich für gleichbedeutend mit $e$ no fim (d. h. nach und trotz so vielen Bemühungen meinerseits). Das Adverb assy weist auf den folgenden Satz hin, ebenso wie an dieser Stelle: o dereilo quer assy que tal poder aia (= haja; Port. mon. hist. I S. 307), und entspricht dem lateinischen sic in: sic a maioribus nostris accepimus praetorem quaestori suo parentis loco esse oportere (Cic.).

Br.: sempr' ouve, poys deus quer assy; que.

15/6 L.: e a gran coita.

$\mathrm{Br}$. behält das handschriftliche a gran coita bei.

16/10 L.: sempr' averey.

Br.: sempre verey.

16/12 L.: averia (die Handschrift hat auia für au' ia).

Br.: avia.

16/13 Monaci's Verbesserung de non falar ist richtig. Mit Verletzung des Sinnes und der Metrik schreibt Braga de non se falar. Augenscheinlich war ffalar die ursprüngliche Schreibart (vgl. Z. 9), und hat der Abschreiber $s$ für $f$, wie noch in anderen Stellen gesetzt.

17/8 Ich lese: Que eu ey d' ela, poi-la vi, levado (ey levado $=$ ertragen habe).

Br.: Que ouv' i d'ela, poil-a vi; levado, was unverständlich ist.

I 7/1 3 Coelho hat die richtige Lesart scharfsinnig herausgefunden. Was Braga statt dessen vorschlägt, ist sinnlos.

17/14 Widersinnig ändert Braga das erste Wort (servo, oder vielmehr servho) in quero.

17/16 Das handschriftliche ouuha leuar (d. h. houv' a levar) ist fehlerfrei. Das $h$ steht für den heutigen Apostroph (Vgl. 5/4, 35/1 7 , I08/1, 235/1 7 u. s. w.) Braga's I.esart punh a levar giebt einen verkehrten Sinn.

18/9 Der Vergleich mit der entsprechenden Zeile in der I. und 3. Strophe zeigt, dals Coelho's Lesart sro sabedor die richtige ist.

Br.: sendo sabedor.

20/3 Ich lese: desy ar ey gram mal, und erkläre: dafür (nämlich dafs ich euch liebe) bekomme ich ein grofses Übel.

Br.: desejarei gram mal.

20/9 Ich lese: desy ar ey mui.

Br.: desejarey meu.

20/14 Das handschr. no munde (d. h. no mund'e) ist richtig $e=$ und doch.

Br.: no mundo. 
22/7, 8 L.: enton a verey; desi sabedor Sro d' atanto (szo nach der vortrefflichen Berichtigung von Coelho). gereimt.

Br.: enton averey desi sabedor. Scond' a tanto durchaus un-

25/3 Ich lese: fazerde-lo (= fazerdes of peyor.

Br.: fezer del o $p$.

25/6 Ich lese: e leixade mim, que vos quero bem.

Br. läfst die hinsichtlich des Sinnes und der Metrik fehlerhafte handschriftliche Lesart et leixade que vos quero bem unverändert.

25/12, I 3 Anstatt des fez Z. I 2 lese ich faz, da das Verbum des entsprechenden relativischen Satzes Z. 5 im Präsens steht; und Z. 13 lese ich praz, was Reim und Sinn genugthut.

26/2 Ohne Zweifel ist Monaci's vortreffliche Konjektur avia anzunehmen, und Braga's Lesart dizia zu verwerfen.

28/I Vielleicht: queixei-m' eu.

Br.: quex' eu m' a vós.

33/I I Wahrscheinlich: porque non vej' a que mi deu.

Br.: porque ceg' a qut mi deu, was keinen annehmbaren Sinn bietet und das Versmals mangelhaft lälst.

33/19 L. ajudar, wie es sich in Tr. $e C$. findet. Braga's Lesart guidar ist schon der Metrik wegen zu verwerfen.

35/2 L.: a mui gram, wie es sich in $\operatorname{Tr}$. $e C$. findet und von der Metrik gefordert wird.

Br.: mui gram.

36/16 Ich lese: de querer sa morte, se.

Br. und Tr. e C.: de q. s. mort', e se, was den Sinn stört.

36/1 8 Beizubehalten ist das handschriftliche com' eu fas' (= wie es auch bei mir der Fall ist), $e$.

Br. und Tr. e C.: com' eu faço.

40/10 Aus der Vergleichung von Z. Io und Z. 4 erhellt es, dafs mays quero-m' al dizer die richtige Lesart ist.

Br.: $m$. $q$. mal d.. was hier durchaus unpassend ist.

4I/6 Der Zusammenhang fordert, meine ich, dals man quem nicht für ein bezügliches Fürwort, wie es Braga scheint, sondern für ein unbestimmtes (- ein gewisser) halte.

$4 \mathrm{I} / \mathrm{IO}$ Es ist keineswegs nötig, den Modus des Zeitworts zu ändern (e servo-vos oder lieber $e$ servho-vos).

Br.: servir-vos.

4I/24 Indem ich für das letzte Wort die vortreffliche Konjektur Monaci's benutze, schlage ich vor zu lesen: ca mui ben, par Deos, eu ando sandeu. In Betreff der Trennung des mui von sandeu vgl. Muyt' ando triste no meu corazon (42/1).

Braga's Lesart ca mim bem perdud' e sandeu hat keinen Sinn und lälst das Vermals unvollständig.

48/14 L.: søo (in Tr.e C., wie gewöhnlich, soon geschrieben). Br. setzt som, sodals der Vers um eine Silbe zu kurz bleibt. 56,'I2 L. mit Monaci: primeiro.

Br.: por mal, was die Metrik selbst verwirft. 
64/13 Das handschr. chari ayras ist gewifs ein Eigenname, vielleicht Johan Ayras.

$\mathrm{Br}$.: charryar-as von dem - unmöglichen Zeitworte charriar. 64/14 Ich lese: Alvelo (welcher Name auch 1079/1 vorkommt). Br.: Alvel' $a$.

66/1 L.: viimos (oder lieber vĩ imos), wie es die Metrik verlangt. Br.: vimos.

$69 / 2$ Nach meiner Meinung ist das handschr. volta (= Verwirrung) e guerra richtig.

Br.: vôlta a guerra.

74/2 Ich lese: estremece. Vgl. 74a/6.

Br.: estremente, ein unerhörtes Wort.

81/1 7 L.: loor.

Br.: teor, unpassend.

87/3 Das von Braga beibehaltene $e$ im Anfange des Verses ist sicher $\mathrm{zu}$ unterdrücken.

89/9 Ich lese: e pois me Deos non val.

Br.: pero se me d.n. $v$., sodals er den Vers um eine Silbe zu lang lälst.

92/3 L.: mays ca m' ey (= sondern dafs ich habe) de trobar. Vgl. Z. I4 mays o gram sabor que $m$ ' end' ey, und 58I/IO, I I.

Br.: m. $c$ amei de t.s. Man kann nicht amar sabor sagen.

$97 / 3,7.1$ I Ich lese: veedes.

Br.: vedes.

97/3 Ich lese: quen $e ́$, seede. Vgl. 7,I I.

Br.: quen é, e seede.

100/2 Ich lese: forçar a poder (e für $a$ kommt noch an sechs Stellen vor). schliefst.

Br. behält $e$ bei, was eine sprachliche Unrichtigkeit in sich

I $3 / 4$ Ich lese: $\dot{e}$ (oder est) a molher.

Br.: a molher.

I1 3,6 L.: sempr'e.

Br.: sempre. verlangt.

I $4 / 2$ Ich lese: de vos poder veer, wie es die Sprachrichtigkeit

Br. behält se v. p. v. bei.

I15/1 L.: de.

Br. behält do bei.

I 20/9 Das handschr. amor ist richtig. Es ist das Subjekt des Satzes.

Br.: a mór.

124/1 8 L. mit Moura ajudador.

Br.: avĩ dador.

126/6 Vielleicht: Tanto me coyta e traz mal amor. Vgl. 134/1, 2 Assy me trax coytado | e aficad' amor.

Br.: Tanto m'é coyta e trax i mal amor. 
126/I I Ich lese: atanto.

Br.: e tanto.

129/9 Vielleicht: poss' oj' osmar.

Br.: poss' $i$ osmar.

1 $36 / 17$ Ich lese: terria-me, e razon faria (= und daran würde ich recht thun).

Br.: terria m'en razon, faria.

143/2 L.: poys a que.

Br.: poys que.

143/I 4 Ich lese: nunca (oder jamais) non pud' aver se coita non.

Br.: lärst die fehlerhafte handschr. Lesart non pud' aver se coita non unverändert.

159,2 6 Ich lese: querria ( $i$ für $r$ kommt öfters vor).

Br.: queria.

$160,13,14$ Das handschr. seve (Perfectum von seer) ist richtig.

Br.: s' eve, unerklärbarerweise.

182/1 8 Vielleicht: por (oder lieber pois) que vós perjurastes, amigo. Vgl. das Ende der zwei ersten Strophen.

184/9, 196/1 3 L.: valrria.

Br.: valeria.

196/16 Das handschr. que ist richtig (eu, que non nacesse = ich, die nicht hätte geboren werden mögen). Vgl. 207/3.

Br.: quen.

202/9 Nach meiner Ansicht ist das non zu streichen, welches den Sinn und das Versmals stört.

202/20, 2 I6/0 L.: $e$ (= und).

Br.: é.

224/20 Der Metrik wegen lese ich: e quanto.

225/14 L.: dev'.

Br.: deu.

246;5, 10 Man mufs ler lesen, wie es aus dem Reim erhellt.

Br.: lez (5), lex (10).

246/8 L.: aguardar. Vgl. $\{3$.

Br.: guardar.

249/2 Ich lese: qual est 0.

Br.: e qual he o.

249/4 L.: 0 voss' amigo.

Br.: voss' amigo.

251/3, 4 L. mit Monaci: falar migo; vin eu.

Br.: f. migo sin eu.

252/3 L.: iredes comigu' $i$ ? Augenscheinlich ist es eine Frage.

Br.: $d$ 'iredes comigu' $i$.

253/7 Das handschr. no ( $=$ ihn) ist richtig.

$\mathrm{Br} .:$ nom, ungereimt.

260/2 Ich lese: a meu amigo non th' ouso falar. Vgl. 264/1 A mha coita non thi sei guarida.

$\mathrm{Br} .:$ meu amigo, als ob es ein Vokativ wäre. 
$260 / 3$ Das e amigo ist richtig.

$\mathrm{Br} .:$ cá migo.

279/7 Ich lese: guardey, wie es die Metrik und die Sprachrichtigkeit verlangen.

$\mathrm{Br} .:$ guardarev.

289/ I I L.: $c$ assy lhe praz.

Br.: ca si lhe p. .

297/I5 L: $\operatorname{sen}$ (= ohne, d. h. wäre es nicht).

Br.: s'en, ungereimt.

298/I L.: and'e.

Br.: ando.

300/4 Ich lese: muit' errada ( $c$ für $t$ kommt öfters vor).

$\mathrm{Br}$. behält das handschr. mui cerrada bei.

$300 / 9$ Das handschr. fiz ist richtig.

Br.: fez.

300/14 Ich lese: El de pran que esto cuydo |que est amigo perdudo (oder que está migo perdudo), sodals el das Subjekt zu est sei, und esto auf den Satz que est amigo perdudo hinweise. Hinsichtlich der Stellung des Subjekts el vgl. 327/I, $2 O$ voss' amig', amiga, foi sazon que desejava.

Br.: El de pran quen esto cuyda | que est migo perdudo. 301/5 L.: lo meu (= der meine).

Br.: lo $m$ ' eu, ungereimt.

309/2 Ich lese: soýa a bafordar (soýa das Imperfectum von soer).

Br.: behält soy a abafordar bei.

309/2 I Ich lese: que lh' eu fiz, hu (= damals als; vgl. 269/4) m'a cinta vēo (= lat. vēnit) a cinger.

Br.: que lh'eu fiz huma cinta veu a cinger, unverständlich.

312/10 Ich lese: guarecer.

Br. behält das handschr. couorecer bei, welches unerhörte Zeitwort schon des Versmafses wegen zu verwerfen ist.

328/7 Ich lese: por mi, meu ben $\dot{e}$ de lhi ben fazer (meu ben $\dot{\varepsilon}$ = das ist recht von mir; vgl. den Gegensatz dizem que é mal Z. 3).

Br.: por mi meu ben e de thi b. f., unverständlich.

329/5 L.: poys $m^{\prime}$ el ben quer e que lh' eu faço mal. Das que steht für das poys des vorigen Satzes; vgl. 96/2, 3 I5/4.

Br.: behält faça bei, was den Sinn stört.

335/8, 9 Ich lese: el faz aguisad' (= er thut recht daran); eu po-lo fazer non lh' o gradesco (= ich weifs ihm keinen Dank dafür, dals er so thut). ständlich.

Br.: el faz guisa d' en polo fazer, nem lh'o gradesco unver-

353/1 L.: Morr' o meu amigo d' amor.

Br.: Morro, meu amigo, d' amor, im Gegensatz zu dem allgemeinen Sinne des Liedes.

354/2 por mi non pesar (= um mich nicht in Trauer zu versetzen) non morria ist richtig.

Br.: por mi com pesar non morria, ungereimt. 
362/7 Das talan (apokopierte Form von talante) ist richtig. $\mathrm{Da}$ das Versmals aber mangelhaft wäre, so lese ich: Que prol lhe tem ou que talan ihe dá Vgl. 548/16.

Br.: Que p. tem ou que fala lhe dá.

380/4 L.: morr' (= stirbt) agora. Vgl. Z. Io.

Das handschriftliche por me veer ist richtig.

Br.: por $m$ 'o veer.

$382 / 2,3$ Der Text ist richtig. Man mufs interpungieren: amig' (= mein Lieber!), anda-mi sanhuda mha madre; .. Vgl.4I7/3.

Br.: amiga, andad' a mi sanhuda; mha madre...

393/8 Das handschr. des (durch Abkürzung $\partial s$ geschrieben) ist richtig.

Br.: deus.

394/I Das handschr. dona que eu quero gran ben ist richtig. Vgl. 4 I $2 / 2$.

Br.: don' a que q. g. $b$.

$420 / 8$ Es scheint mir, dafs das handschr. por ren meos (oder lieber méos) de o veer richtig ist, und ich fasse das meos de im Sinne des Französischen à moins de.

B r.: pero tem meos de o veer.

428/4 Man muls mit den Tr.e C.: direi-vo-lo lesen, wie Grammatik und Metrik verlangen.

B r.: $d y r$-vol-o.

444/I 2 L.: e pero que sey que lh' est' (= dies) é muy greu, da das Versmals sonst mangelhaft wäre und die Form est $=$ lat. est nur vor Vokalen gebraucht wird. unverändert.

Br.: lälst das handschr. e pero que sey que lh' est muy greu

445/1 4 Das handschr. vou ist richtig.

$\mathrm{Br}$.: com, ungereimt.

450/1 8 L.: sol (= lat. solet) Deus.

Br.: sol des, unverständlich.

$457 / 17$ Das cousecem $m i$ ist richtig.

Br.: couseg' en min.

$457 / 18$ Das sy $(=$ sich $)$ ist richtig.

B r.: s'y.

$463 / 2$ Das prol ist richtig.

$\mathrm{Br} .:$ pela.

463/4. 5 Man mufs nicht den Text ändern: e a senhor por que mh assi matades / al cuida ca non no vosso cuydar. Noch jetzt ist que nao (= und nicht) gebräuch lich.

Br.: ca, senhor, porque 'm' assi malades | al cuid' acá, nom no vosso cuydar, was Niemand verstehen kann.

479/30 Das handschr. graves ist richtig.

Br.: grandes.

482/ I5 Das handschr. poder $e$ ist richtig, so dafs man lesen mufs: poder e sen (treffende Konjektur von Monaci).

Br.: perder o sen, ungereimt.

Zeitsohr. f. rom. PhII. XI. 
$483 / 7$ Ich lese: $d$ ' homen.

Br.: de 'm'eu.

493/5 L. d'aquend'ir (aquende : aquem = porende : porem).

B.: d' aquem d'ir.

494/3 Ich lese: eu de mi ben cuydav' enton.

$\mathrm{Br}$. behält die unpassende handschriftliche Lesart bei.

495'7 Der Text ist richtig: Quisera-m' eu.

Br.: Quiçá m' en, ungereimt.

498/1 8 Die handschr. Lesart non sey eu ist richtig.

Br.: non sey, ou.

499,'4 L.: nacêra.

Br.: nacerá.

$501 / 2$ Die handschr. Lesart $e o$ ist richtig.

Br.: c'o gegen Metrik.

514/7 L.: Quemquer que.

Br.: Que quero quem, gegen Metrik und Sinn. $029 / 2$.

523/1 Ich lese: o que me foy miscrar (= frz. brouiller). Vgl. werfen.

Br.: o que me faz mister, schon wegen des Reimes zu ver-

529/1 5 L.: tenh' $y$.

Br.: tem hy.

531 ;3 Vielleicht: levo (handschr. se no).

Br.: se nom, durchaus unipassend.

Das handschr. $e a$ ist richtig.

Br.: cá.

538/5 Vielleicht: pod'o mentiraz (= Lügner). Hinsichtlich des Suffixes vgl. trapaz (oder lieber trampaz) 14/5.

Br.: pode mentir al, ganz und gar unpassend.

540/1 2 L. querrey.

Br.: querey.

542/7 Ich lese: hir-m' eu ( $y$ für $u$ kommt auch $439 / 14$ vor).

Br. behält hir-m' $e y$ bei.

$542 / 15$ Nach der treffenden Konjektur von Monaci ist morreredes zu schreiben, wie die Metrik verlangt.

$\mathrm{Br}$. behält morredes bei.

543, I L.: e de.

Br.: de.

544/2 L.: quizess', e.

Br.: quizesse,

544/I 5 L.: passey-os. Vgl. 3, 9.

Br.: passê-os.

545/4 Ich lese: faça viver, wie Metrik und Grammatik verlangen.

Br.: fez viver.

545/8 Das handschr. fezer (mit Abkürzung $f e z$ ') ist richtig.

Br.: fez. 

$a$ vor.

547/2 Ich lese: ando. Noch an zwei Stellen kommt $q$ für

Br.: que do, ungereimt.

548/1 4 L.: amor. Vgl. 20.

Br. behält maior bei.

548/2 I Ich lese: que vos faria de grad' ess' amor (= Wunsch). Br.: q. v. f. de gram desamor.

550/7 Ich lese: part'o coraçon.

Br.: perc'o $c$.

553/8 L.: fiador.

Br. behält fidor bei.

555/I3 L.: rog 'a Deos que m'ajud' e mi valha.

Br.: rogades quem $m$ ' ajud' $e$ mi valhu, ungereimt.

559/3 L.: essa mi faz a mi peyor.

Br.: e ss' a mi f. a. m. p.

56o'5, 6, 7 L.: e poys a vós aquest' (= dies, näml. dals ich euch liebe) $\dot{e}$ greu, greu vos servi", a meu cuydar (= nach meiner Meinung), d'amardes mi.

Br.: $e$ poys a vós aqueste greu vos seria meu cuydar d' amardes mi, unverständlich.

$560 / 19$ Das handschr. perco (= ich verliere) ist richtig.

Br.: pero.

564/1 2 Wahrscheinlich: d'en mi pensar.

Br.: de mi pensar.

566/26 L.: que Deos guarda.

Br.: que desguarda.

576/22 Ich lese: chamou.

Br.: $x$ ' o amou.

579/8 Ich lese: e o dormir, wie die Metrik verlangt.

Br.: e dormir.

580/4 L.: sey já. Vgl. 10.

Br. behält sey la bei.

583/8 L. mit den Tr. e C.: pero m' eu viv'.

Br.: per omen viv'.

585/5 L.: eu, meus amigos. Vgl. II.

Br.: eu, amigos.

585/8 L. mit den Tr. e C.: coit' em que.

Br.: coita que.

596/8 Ich lese: de sa prol.

Br.: d' essa $p$.

$598 / 7$ Das handschr. que ist richtig.

Br.: quem.

599/2 L.: que vos veja esse fustam trager.

Br.: q. v. v., se f. trager, gegen die Metrik, die Sprachrichtigkeit und den Sinn.

599/3 Vielleicht: a todo vosso poder.

Br.: $e$ tod' $a$ v. p.

616/10 Der Metrik wegen füge ich ar vor falar hinzu. 
620/20 L.: quiser.

Br.: quer.

62 I/ I L.: Diz. Vgl. 3, 10, 12, 15.

Br.: Finge.

625/16 Die vermutliche Lesart von Monaci non mi quer ist dic richtige.

Br.: non quer.

627/5 Augenscheinlich ist zu lesen: queyramos.

Br.: queyremus.

628,8 L. morreredes, wie die Metrik fordert.

Br.: morredes.

629/r Ich schlage vor, zu lesen: Alguem vos diss', amig', e seio eu. Vgl. 636/16, 17. verstehen kann.

Br.: Amigu', eu vos diss' amigo, e serio eu, was Niemand

632/8 Das handschr. sanha, wofür Braga ungereimt senhor setzt, ist richtig.

670/16 L.: direi-vos, wie der Zusammenhang und die Vergleichung von 3 und $\mathrm{I} 3$ fordert.

$\mathrm{Br} .:$ diremos.

$646 / 7$ Es scheint mir richtiger, deu zu lesen und das eu in ro nicht $\mathrm{zu}$ verwerfen, als mit Braga das dá zu behalten und $c a ́$ in Io zu lesen.

646j15 L.: de tod' esto.

Br.: tod' esto.

- 647/13 L.: o mui.

Br.: em mui.

648/14 Vielleicht: pero vivia, nunca vi prazer.

Br.: pero vi, já nunca vi prazer, was keinen befriedigenden Sinn bietet, und den Vers um eine Silbe zu kurz läfst.

656/14 Augenscheinlich muls man lesen: partir (= aufhören) de vos já sempre querer ben. Vgl. 1044/1 2, I 3 .

Br.: partir de vós já sempr' e querer bem.

663/6 L.: $x$ est a mha coyla mayor.

Br.: $x$ ' esta.

663/7 L.: fạ̧' en.

Br.: faz em.

663/28 Man mufs melhoria beibehalten.

Br.: melhor já.

667/13 Monaci's vortreffliche Verbesserung olhos no mund' ond' $e u$ ist unzweifelhaft zu genehmigen.

Br.: olhos, no mund' eu.

675/8 L.: í no.

Br.: e no.

675/1 2 L.: quereria, wie es die Metrik verlangt.

Br.: queirya, was eine falsche Form ist.

688/23 Vielleicht: quem na tan muyt' amava.

Br.: quem nom t. m. a. 

ist richtig.

697/12 Das handschr. ben fis estou (= wohl gewifs bin ich) Br.: 6. fis entom.

698/3 L.: e devo m' eu d' est' a maravilhar. Das Fürwort esto weist auf den folgenden substantivischen Satz hin. Vgl. 300/13, 14.

Br.: e devo m' eu d' esta maravilhar.

700/9 Das vatikanische $e$ a $m i$ ist richtig.

Br.: ca mi.

704/14 L.: que ha hum ann' ou mays. Vgl. die der 13: und I4. entsprechenden Zeilen 20, 21 .

Br.: que a hum, e nom mays, was keinen Sinn hat.

708/25 Ich lese: atâm. Das Adverb atão, eine apokopierte Form von atanto, wie tao von tanto, kommt auch 708/18, 787/1 2 vor, in welchen Stellen Braga fälschlich $x$ tam schreibt.

Br. ganz unpassend: $a$ fame. zu sein.

717/9 Monaci's Lesart adur diria scheint mir die richtige

Br.: dizer temia.

729/1 3 L.: muyt' al.

Br. gegen Sinn und Metrik setzt muyt' a mal.

733/2 L.: faledes, wie die Grammatik verlangt. Vgl. tardades für lardedes $730 / 11,17$.

Br. behält falades bei. ao lesen.

Für das do, welches Braga beibehält, mufs man mit Monaci

763/1 L.: fez Deus a.

Br.: fiz, Deus, a.

763/8 L. mit Monaci: se a ela praz (s'a ela praz, um der Netrik willen).

Br.: se ca l'apraz.

L.: de thi.

Br.: de $l h^{\prime} y$.

77 I Das manh (= lat. maneo) eu Z. I, und das masesse (= lat. mansissem) Z. 5, I I, I6 sind richtig. Braga, der jenes antiquierte Verbum nicht zu kennen scheint, schreibt Z. I m' and' eu Z. 5, I I, $16 m^{\prime}$ a desse. Auch 1049/4 ändert er das richtige masestes (= lat. mansistis) in das unbegreifliche massastes. zu sein.

793/I 8 Monaci's Lesart muyt' amava scheint mir die richtige

B.: muyt' a vós ama.

818/10 Das e ide-lhi dizer ist richtig.

Br.: et de lhi dizer.

$822 / 15$ Das que vos disser ist richtig.

$\mathrm{Br} .:$ quem v. $d$.

822/19, 20 Ganz fehlerfrei ist der vatikanische Text: e achouas partir todas d' amor (d.h. und er fand, dafs sie alle der Liebe 
nicht treu bleiben): In Betreff der Wortfügung vergleiche man Z. 9, 10 achey-o jazer sen fala.

Br.: $e$ achou, ao partir todas d' amor.

823/4 L.: porque, filha (= Tochter), desque o vós conhocestes.

Br.: porque filhades que o v. c.

837/1 5 Dem Scharfblicke Monaci's ist es nicht entgangen, dafs nach seu das Wort poder fehlt.

Br.: seu ser.

840/10 L.: atant' entendem mays. Das atanto mays ist korrelativ dem quanto mays in der vorigen Zeile. $\mathrm{zu}$ verwerfen.

Braga's Lesart al entendem mays ist schon der Metrik wegen

850/6 L.: donde.

Br.: dor ende, unverständlich.

853/1 Monaci's hypothetische Lesart pois scheint mir die richtige zu sein.

863/10 L. mit Monaci perdedes, wie es die Metrik verlangt.

Br.: perdês.

870/1 2 Vielleicht: est' $e$ lo me que mays demandava (= das war das Grölseste, das er von mir verlangte).

Br.: est'é l'ome q. m. d.

$887 / 13$ e muls in son korrigiert werden, wie es von der Syntax verlangt wird und sich $Z .8$ findet.

Br.: behält $\ell$ bei.

900/5 L.: olhos. Vgl. 10, 15.

Br.: $d$ ' oos, unverständlich.

907/I L. Rodriguiz. Roiz ist eine noch jetzt gebräuchliche Abbreviatur von Rodrigues.

Br.: Roiz. Ebenso 1037/1.

907/4, 5 Vielleicht: nunca s'a el chegou, nem quer chegar, se d'el certa non he.

Br.: nunca ss'a el ch., $n$. q. chegar-se d' el; sancta non he.

909/1 7 Ich glaube, dafs porrei eu die richtige Lesart ist; diese Zeile' soll ja denselben Gedanken enthalten wie die Zeilen 5 und I1. An zwei andern Stellen kommt noch $z$ statt $y$ vor.

Br.: pareceu.

916/3,4,5 Ich lese: em no parecer (= Miene) entender pode, quant' $\&$ mha creença (= wie ich glaube), que. ständlich.

Br.: e non parecer entender porquant' é mha c., que unver-

916/9 L. mit Monaci feituras.

Braga liest: feyestas, und erklärt: festas. Nach den Gesetzen der romanischen Phonetik ist eine Form wie feyesta neben festa nnmöglich. Auch wäre solch ein Begriff hier unstatthaft.

916/I I Der Text ist richtig. quant' $e$ meu ciente ist gleichbedeutend mit quant' é mha creença Z. 4 .

Br.: quant' $e$ menciente (= mentiroso nach Braga).

$921 / 18$ L.: guarecer. 
Br.: guarrer, was schon der Metrik wegen zu verwerfen ist. 940/2 Man mufs interpungieren: era; mentia.

Br.: ora mentia. richtig.

977/18 Das handschr. carcer atal (= solches Gefängnis) ist

Br.: cerc' a tal.

98I/I I Das handschr. bra coor ist richtig.

Br. schreibt bon coor, als ob das zweisilbige coor (= colorem) das lat. cor repräsentieren könnte.

981/14 L.: pareceredes, wie die Metrik und der Sprachgebrauch verlangen.

Br.: parecedes.

989/1 6 Ganz richtig sind die Textesworte como alberguei, non albergaria ( = ich würde nicht so geherbergt haben, wie ich geherbergt habe).

Br.: como alberguei na albergaria, als ob das albergaria hier ein Hauptwort wäre.

992/4 Das handschr. sorrabedes ist richtig. Es ist der Konjunkt. des Präs. von sorrabar, einem Abgeleiteten von rabo. Hinsichtlich des Sinnes vgl. Z. II.

Br.: saberedes.

IOI3/1 toruado (d. h. torvado = lat. turbatus) ist richtig.

Br.: tornado.

1047/1 2 Ich lese: se $x^{\prime}$ est' (= dies) é carne, se pescaz. (In Betreff der Hinzufügung des reflexiven Fürwortes zum Zeitworte ser vgl. 663/6).

Br. schreibt: se $x^{\prime}$ est carne, se pescaz, sodals der Vers mangelhaft bleibt, und est für das Präsens vom Zeitwort ser gelten soll. Die Form est aber kommt nur vor Vokalen vor.

1069/1 2 Ich lese: $e$ pois aqui vee[r] la invernada = und wenn die Winterzeit hierher gekommen ist.

Br.: e p. a. veél-a n' invernada.

I $100 / 4,5$ Der Text ist richtig, aber Br. macht ihn unverständlich, indem er en $a$ anstatt des $e \tilde{u} a$, und pois anstatt des pos (=- lat. posuit) schreibt.

I I 66/6 Richtig ist jaredes, eine verkürzte Form von jazeredes, ebenso wie diredes, faredes.

Br.: jazedes, gegen den Sinn (vgl. Z. 8).

Epiphanias Dias. 